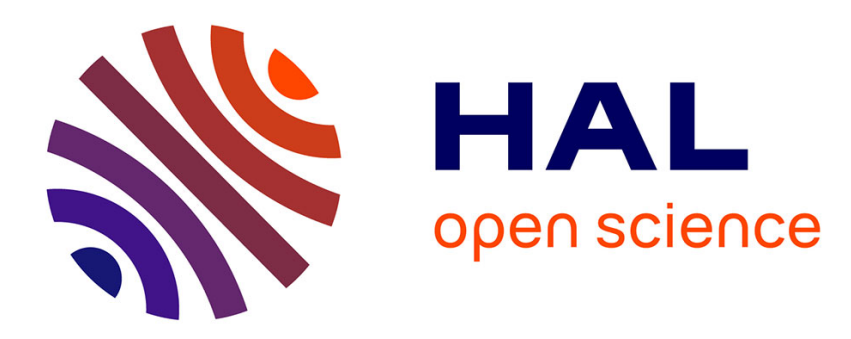

\title{
Les infortunes de " notre" amour de la vérité face à l'emprise du principe de plaisir
}

François Villa

\section{To cite this version:}

François Villa. Les infortunes de " notre " amour de la vérité face à l'emprise du principe de plaisir. Cliniques méditerranéennes, 2004, 70 (2), pp.91 - 111. 10.3917/cm.070.0091 • hal-01496627

\section{HAL Id: hal-01496627 \\ https://hal.science/hal-01496627}

Submitted on 27 Mar 2017

HAL is a multi-disciplinary open access archive for the deposit and dissemination of scientific research documents, whether they are published or not. The documents may come from teaching and research institutions in France or abroad, or from public or private research centers.
L'archive ouverte pluridisciplinaire HAL, est destinée au dépôt et à la diffusion de documents scientifiques de niveau recherche, publiés ou non, émanant des établissements d'enseignement et de recherche français ou étrangers, des laboratoires publics ou privés. 


\title{
LES INFORTUNES DE « NOTRE » AMOUR DE LA VÉRITÉ FACE À L'EMPRISE DU PRINCIPE DE PLAISIR
}

\author{
François Villa
}

\section{ERES | « Cliniques méditerranéennes »}

2004/2 $\mathrm{n}^{0} 70$ | pages 91 à 111

ISSN 0762-7491

ISBN 2749202701

Article disponible en ligne à l'adresse :

http://www.cairn.info/revue-cliniques-mediterraneennes-2004-2-page-91.htm

\section{Pour citer cet article :}

François Villa, « Les infortunes de « notre » amour de la vérité face à l'emprise du principe de plaisir », Cliniques méditerranéennes 2004/2 (nº 70), p. 91-111. DOI 10.3917/cm.070.0091

Distribution électronique Cairn.info pour ERES.

(C) ERES. Tous droits réservés pour tous pays.

La reproduction ou représentation de cet article, notamment par photocopie, n'est autorisée que dans les limites des conditions générales d'utilisation du site ou, le cas échéant, des conditions générales de la licence souscrite par votre établissement. Toute autre reproduction ou représentation, en tout ou partie, sous quelque forme et de quelque manière que ce soit, est interdite sauf accord préalable et écrit de l'éditeur, en dehors des cas prévus par la législation en vigueur en France. Il est précisé que son stockage dans une base de données est également interdit. 


\section{François Villa}

\section{Les infortunes de «notre » amour de la vérité face à l'emprise du principe de plaisir 1}

... Un ange !... Chut !... Un ange passe ! L'avez-vous vu ?... Moi... Je l'ai vu. Et, terrorisé... je me suis tu... pour ne pas attirer l'attention de l'ange. Ce n'est pas le désir qui m'en manquait, j'ai simplement manqué de courage. Je l'ai donc laissé passer sans mots dire, mais en pestant in petto. Je n'ai pu agir autrement. Non pas parce que l'expérience m'a appris qu'on invoque toujours en vain un ange, en effet, ne connaissant pas l'arrogance (qualité moïquement humaine), les anges ne dédaigneraient pas de nous répondre. Un ange... patiemment - il a l'éternité devant lui - attend $\mathrm{qu}^{\prime}$ « un n'importe quoi qui d'aventure vient à se présenter 2 » dans la vie d'un homme provoque son évocation et, aussitôt, de manière fulgurante, directe et immédiate, il est, indiscutable présence.

Déjà, je regrette de l'avoir laissé passer. Et cela bien que je sache qu'en m'abstenant, je l'ai sans doute échappé belle, car, à tout coup, j'aurais été convoqué là où il n'est plus possible d'accorder à moi ni l'intérêt auquel il prétend avoir droit, ni l'importance qu'il voudrait se voir reconnaître.

François Villa, psychanalyste, maître de conférences à l'université Paris 7-Denis Diderot, 30 bd de Strasbourg, F-75010 Paris.

1. Cet article est la reprise remaniée d'une conférence donnée au $4^{\mathrm{e}}$ Groupe le mercredi 11 juin 2003. Je tiens à remercier plusieurs collègues et amis : d'abord, G. Lévy qui m'a donné l'occasion de faire ce travail et qui a accepté d'en être le discutant, ensuite N. Zaltzman qui est une interlocutrice précieuse et enfin C. Cyssau qui, tout en m'incitant à suivre la piste de l'ange, a veillé à ce que l'amour de la vérité ne subisse pas trop d'infortunes. Dans ce texte, est présent $\mathrm{P}$. Fédida dont j'éprouve la cruelle absence.

2. Jean de la Croix, "Glose "a lo divino" ", dans CEuvres complètes, trad. par le P. Cyprien de la Nativité de la Vierge, Paris, Desclée de Brouwer, 1967, p. 931-932. 


\section{L'ANGE, LE TERRIBLE ET LA DÉSIRANCE}

Un ange... "Tout ange est terrible », c'est Rilke qui l'écrit: "Jeder Engel ist schrecklich ${ }^{3}$ ». N'ayons pas le moindre doute, le mot est bien "schrecklich 4 », et c'est bien de l'effroyable dont il est et sera question. Car, l'ange n'est pas angélique. L'angélisme 5, cette humaine négligence de la réalité, lui est inconnu. Un ange prête attention au plus petit détail, à l'apparemment insignifiant, sachant que ce n'est que par ce truchement que parfois l'humain s'ouvre enfin, à nouveau, à ce qui le meut. Oui ! l'ange est terrible! d'autant plus terrible qu'il arrive que, passant, il y en ait un parmi les hiérarchies des anges qui, alors que manifestement nous n'avions rien demandé, nous prenne sur son cœur et qu'accomplissant sa fonction, il nous délivre un message qu'il nous signifie alors même qu'il est pour lui sans signification. Pour l'ange, la question du sens n'est qu'un problème topique. Il va et vient entre deux mondes, vers l'un ou vers l'autre. Son seul souci, et est-ce d'ailleurs un souci, est de se délivrer de ce qui l'a poussé au mouvement. "Qu'est-ce que ça veut dire ? » n'est pas une question d'ange, c'est une interrogation humaine.

L'ange, à qui, à notre connaissance, nous n'avions rien demandé, nous a pris sur son cœur. Moment de grâce, du moins voudrions-nous le croire, comme si nous aimions tant que ça le commerce avec les anges, soient-ils musiciens. Mais voici que, déjà, au cœur même de la joie qui explosant saisit, commence le terrible dont nous ne parviendrons plus à nous exonérer malgré nos peineux et tragiques efforts. Comme il est venu, l'ange se retirera. On ne pourra même pas lui reprocher de nous avoir abandonné, car il n'est tout simplement pas dans la nature de l'ange de se fixer une fois pour toutes - à cela tout ange répugne. L'idée d'avoir, en propre, un ange gardien est un étrange souhait humain car l'ange nous expose bien plus qu'il ne nous garde. Et, il est loin d'être certain que nous ayons envie de le retenir. Par l'histoire de nos pères, nous savons qu'un ange est le plus souvent porteur de messages qui, au mieux, nous paraissent incompréhensibles ou absurdes - ceuxlà, au moins, nous permettent de remettre la compréhension à plus tard ou de les rejeter - mais il en est d'autres, ce sont les pires, dont les conséquences s'imposent à nous insupportables, impossibles à accepter mais tellement impérieuses que c'est vainement que nous essayerons de leur échapper.

3. R.M. Rilke, Les élégies de Duino, trad. A. Guerne, Paris, Le Seuil, coll. «Essais », 1972.

4. P.L. Assoun, "De l'effrayant à l'effroyable. Figures freudiennes de Schreck », dans Apertura, $\mathrm{n}^{\circ} 17,2002$, Toulouse, érès, p. 31-43.

5. Aux anges, l'angélisme est inconnu, c'est une prétention humaine que de vouloir négliger les réalités, non pas que la réalité intéresse les anges, si elle les attire c'est que seule par elle peut s'effectuer leur présence. 
Un ange est passé... Mais, même quand il ne fait que passer, l'ange produit un effet, pour ne pas dire un affect. En passant, il éveille immanquablement non pas la nostalgie, mais la désirance qui est antérieure à la nostalgie. Car, le terrible est que nous ne désirons pas quelque chose que nous aurions été ou que nous aurions perdu, mais ce dont la nécessité se fit paradoxalement sentir à l'orée de notre vie : nous désirons ce qui aurait dû être pour que ça se termine au commencement.

L'ange éveille en nous le primum movens. En raison de sa nature, comme Wim Wenders nous le fait percevoir dans Les ailes $d u$ désir, l'ange est animé d'une perpétuelle désirance d'humanité. Cette "Sehnsucht ", si elle n'est pas pure (la pureté est une préoccupation humaine) est pourtant pure désirance, élémentaire mouvement non pas tant vers un objet que vers l'abolition du mouvement même. À celui-ci, l'objet n'est certes pas nécessaire, mais il lui est pourtant indispensable 6 pour s'abolir comme mouvement. La "Sehnsucht » n'est jamais que la source d'un projet, le premier, qui, pourtant, ne survient que secondairement. Ce " projet 7 » émergera là où " ça » exige que la chosemême soit et s'accomplisse et où est faite l'expérience de l'absence : absence d'une complète satisfaction, absence d'objet, absence de moyens - la pure absence. Cette absence-là est d'une autre nature que celle qui fut précédée $\mathrm{d}^{\prime}$ une présence ${ }^{8}$. En elle, s'éprouve la nécessité vitale de la présence de quelque chose dont nous n'avons pas la moindre idée. La seule idée (qui en est à peine une : une préconception d'idée) que nous en ayons est celle de son impérieuse nécessité. Quelque chose comme « ça » doit nécessairement exister quelque part, Dieu, seul sait où. En cet instant, nous découvrons que nous sommes tenu à et par l'impossible ${ }^{9}$ et que, notre vie durant, nous serons dans la nécessité de faire face à celui-ci pour soutenir ce qui nous tient. La nécessité, comme nous le rappelle l'étymologie, s'éprouve dans l'expérience de $l^{\prime}$ « impossibilité de se mouvoir ou d'être mû », de l' "impossibilité ou de s'en aller ou de reculer » et dans le vécu d'un état de "misère, pauvreté ».

La présence de l'ange est si forte que nous oserons à peine dire qu'elle nous émeut. Elle nous commotionne et engendre une motion qui n'aura d'abord que l'ataxie pour réponse. Certes, en cet état, doit être reconnue la source de toute la palette des émotions humaines, mais nous n'avons pas encore trouvé les moyens pour donner à cet état la qualité d'expression qui

6. Je remercie J.-C. Lavie de m'avoir aidé à repérer plus précisément la distinction qu'il s'imposait de faire entre le nécessaire et l'indispensable.

7. Projet d'où se créeront l'objet et l'intérieur à partir de ce qui ne peut pas être projeté. Cf. S. Ferenczi, «Transfert et introjection », dans Psychanalyse 1, CEuvres complètes : 1908-1912, trad.

J. Dupont avec coll. de Garnier Ph. Paris, Payot, 1945, p. 93-125.

8. Pierre Fédida, L'absence, Paris, Gallimard, 1978.

9. Lacan a avancé que l'impossible était le réel même. 
est celle des émotions. Là, où « ça » aurait dû avoir lieu et où «ça » n'a pas eu lieu, va se produire une différenciation porteuse d'une rare aptitude à différer. La différenciation dont je parle n'est autre que celle d'avec le « ça » qui se produit dans la précipitation 10 où se forme le moi avec la théorie d'objets qui l'accompagnent tout autant qu'ils le composent. C'est ainsi qu'advient cette forme qui, pour obtenir la paix pulsionnelle, est présentée au ça comme la chose même. Mais le ça, intraitable, ne veut ni se conformer avec cette illusion synthétique, ni y reconnaître de l'indispensable.

Et pourtant, ni du moi, ni de son cortège d'objets, nous ne pourrons faire l'économie pas plus qu'ils ne nous permettront de faire l'économie du point de vue économique. Pour approcher du nécessaire, les objets (à commencer par le moi) sont indispensables à la pulsion : condition de possibilité même de tout trajet pulsionnel. Oui, j'ai bien dit de tout trajet, qui pourrais-je bien vouloir outrager? Mais, n'est-ce pas le pulsionnel qui est l'outrage même ? Il semble toujours l'être pour le moi, pour la conscience, pour les convenances et pour les nobles idéaux. Les objets nous sont donc indispensables. Mais, de cela nous pouvons être conduits à tirer trop rapidement la conclusion qu'ils nous sont nécessaires, nous confondons alors le but avec le chemin.

\section{À NOUVEAU, UN ANGE PASSE, L'EXIGENCE D'HABITER LE SILENCE}

Surpris par le cours de mon écriture, je marque une pause, je pressens que plane la menace que la promesse faite dans le titre ne soit pas tenue. Pris par la figure chatoyante de l'ange, n'aurais-je pas confondu le chemin avec le but au point d'oublier le but annoncé par mon titre? Je ne sais pas comment continuer. Je suis troublé, à une croisée des chemins.... À nouveau, un ange passe... Vais-je me résoudre à tenir, autant que je le pourrai, ce que mon titre promettait ou vais-je continuer à suivre l'ange et à me laisser conduire par lui je ne sais trop où?

Ne suis-je pas de fait en train de mettre en œuvre le titre de cet article en faisant subir quelques infortunes à "mon » amour de la vérité. Au travers de la figure de l'ange, le principe de plaisir n'aurait-il pas trouver un moyen de maintenir sa domination et de détourner, autant que possible, ma pensée de certaines idées qui, venues à l'esprit, ne cesseraient d'en être chassées, car malgré l'intérêt qu'elles suscitent en moi, elles sont porteuses d'une charge de déplaisir qui semblent les vouer à un interdit de séjour prolongé dans la conscience. Certes, elles ne peuvent pas être durablement exclues, elles ne le sont que, par intermittence, dans un jeu de da-fort-da..., là - plus là - à nou- 
veau là et, ainsi suite, de manière répétitive, mais ni monotone, ni monocorde. Ce va et vient est cause d'une certaine agitation : un trouble cacophonique de la pensée devenant, du point de vue du principe de plaisir, trop polyphonique. Mais, une pensée qui n'est pas capable de supporter, un peu, le déplaisir que lui causent certaines idées ne peut pas penser effectivement. Nous ne pouvons pas complètement ignorer, même si nous tendons à le négliger, que la pensée ne saurait échapper à ce qui est l'une de ses premières fonctions et, probablement la raison même de sa constitution : nous permettre, en premier lieu, de dépasser une expérience originaire de douleur, du terrible - le Schreck évoqué précédemment ; ensuite empêcher son retour en anticipant tous les signes qui l'annonceraient, et enfin supprimer autant qu'il se peut le déplaisir. Nous ne pensons pas par plaisir de penser, mais par nécessité de le faire. Même, s'il est vrai que nécessité faisant loi, nous parvenons à tirer quelques notables bénéfices secondaires de ce joug que nous subissons inexorablement. La pensée, paradoxalement, ne vise, quant au fond, que l'abolition de la pensée 11.

En prenant pour point de départ la formule « un ange passe », ce n'est pas tant sur l'ange que sur le silence que je voulais attirer l'attention. Et, c'est comme si j'avais oublié le sens de cette expression pour me focaliser sur une partie de cette métaphore, au point d'en oublier le silence en ne parlant plus que de l'ange. Serait-ce extrêmement difficile de parler du silence ? N'est-il pas, peut-être, bien plus difficile que nous ne le reconnaissons de faire véritablement silence et $\mathrm{d}^{\prime} \mathrm{y}$ rester autrement que comme dans un refuge, sous un masque ? Un silence ne pourrait-il pas être parfois la meilleure façon de masquer le silence, comme la logorrhée malgré le flot de paroles qu'elle charrie s'avère être une parole mutique?

Le moment où un ange passe ne serait-il pas très singulier ? Que survient-il à cet instant-là ? Le silence peut durer longtemps, il peut s'installer durablement jusqu'à résonner comme un silence de mort. Il peut être à couper au couteau, pesant, ennuyeux. Il peut être parlant, bruissant de paroles qui, quoi que non prononcées, sont cependant bien entendues. Il peut aussi devenir l'expression d'une loi que l'on dit être celle du silence. Mais, le plus souvent, on ne se laisse pas très longtemps envahir par le silence, on ne le laisse pas s'installer : il y a presque toujours quelqu'un qui le brise et qui prend la parole. En tout cas, même quand le silence dure, la pensée reste rarement vide, elle se remplit, le plus hâtivement possible, de quoi que ce soit, plutôt que d'affronter le silence, de se laisser habiter par lui en l'habitant.

11. S. Freud (1896), «Esquisse d'une psychologie scientifique», dans La naissance de la psychanalyse, trad. A. Berman, Paris, PUF, 1956. 
L'habitation du silence ne ferait du silence un habitat que lorsque « j'habite ma douleur » (R. Char). Habiter quelque chose est d'une autre nature qu'être habité par cette chose. Par rapport à ce qui, nous habitant, nous détermine, nous pouvons soit le reconnaître en surmontant nos résistances, soit vouloir l'ignorer quoi qu'il nous en coûte.

Dans une séance, il n'est pas facile de supporter les formations psychiques et corporelles bizarres qui peuvent émerger dans le silence, de les supporter jusqu'au moment où nous pourrons les habiter en leur donnant des noms qui nous permettront de les habiter en nous (être l'hôte de l'hôte). Ces noms, il nous faudra aussi en suspendre la profération, laissant le patient saisir la chance, que lui aura donnée notre abstinence, de dire, lui-même, les mots qui seront les noms désignant autrement que les nôtres la chose dont ni le patient, ni l'analyste n'auront jamais été si proches qu'à cet instant où ils se révèlent radicalement éloignés l'un de l'autre. Se taire, c'est accepter de prendre la mesure de tout ce que notre parole peut vouloir taire en disant. Il est, par contre, des fois où il nous faut sentir que notre abstinence devient un refus d'assistance et que le moment opportun est venu où nous devons proférer les mots sur lesquels le patient prendra appui pour que des noms viennent en lui.

\section{DON DU ET DE SILENCE}

J'ignorais que j'avais l'intention de parler autant de l'ange - et cela même si Les élégies de Duino de Rilke sont un de mes livres de prédilection, ou, en détresse, j'ai, plus d'une fois, pu retrouver des ressources pour reprendre haleine. En évoquant la formule " un ange passe », je me rappelais un sketch de R. Devos qui, à chaque fois que je l'ai entendu, m'a plongé dans un rire de sidération. Mon projet était d'interroger ce que j'appellerai « un don de/du silence ». L'ange n'étant qu'accessoire, pas nécessaire et même pas indispensable. Conformément à sa nature, il ne devait être qu'un intermédiaire. Aurais-je cédé à une tentation esthétisante dont je ne peux m'empêcher de penser qu'elle me ferait manquer mon but, pour produire par l'écriture ce qu'on pourrait appeler « un bel objet », en la figure de l'ange ?

Peut-être nous faut-il revenir à la question de l'effroi pour entendre la nature du mouvement esthétique. Bien que j'ai indiqué que l'ange était directement en rapport avec l'effroi, j'ai été amené à traiter sa figure de manière plutôt chatoyante, séductrice, voire séduisante. Ne doit-on pas penser que cette manière esthétique de le traiter vise à procurer un gain de plaisir dans le temps même où l'on tente de refouler l'effroi ? Mais, on ne se débarrasse pas si aisément du Schreck. Qu'on le veuille ou pas, il est la force qui habite 
le mouvement d'écriture, alors même que la forme de l'écriture tente de l'expulser. J'écrivais à partir et contre le Schreck, pour essayer de le surmonter.

Nous avancerons que le surgissement du silence est une menace pour la tyrannique domination du principe de plaisir, un moment où son au-delà risque d'émerger en laissant entendre tout ce dont le principe de plaisir tente de nous prémunir. Le silence n'est-il pas l'une des formes les plus fortes et les plus expressives de la manifestation de la compulsion de répétition ? N'estil pas un moment particulier où cette pulsion de mort, dont Freud dit qu'elle œuvre silencieusement, cesse d'être silencieuse pour se faire intensément parlante dans ce blanc de la parole qu'est le silence ? Par prudence et parce que je me méfie de la précipitation du moi à parvenir, à tout prix, à une synthèse, je me garderai d'affirmer que cela est vrai pour le silence en général. Ce n'est peut-être défendable que pour certains types de silence. Ce sont ceux-là que j'appelle don du et de silence.

La non-réception de ce don a pour effet un appel de et du transfert qui est précipitation dans la répétition des solutions qui se sont avérées soit conformes à ce principe, soit de celles permettant de le réinstaurer quant il s'est vu menacé par la répétition de ce qui se révèle au-delà du plaisir. Mais, ne suis-je pas de nouveau, en train de tomber sous l'emprise de ce principe de plaisir ? En disant que ce don est appel du et de transfert, je néglige qu'il ne l'est que secondairement. L'appel du et de transfert est un contre appel, le transfert est appelé, à la barre, contre... Oui, contre, mais contre quoi donc ? Plusieurs noms me viennent pour désigner cette chose, certains, je les ai déjà employés au cours de ma dérive angélique : le terrible, l'effroi, l'impossible, le nécessaire. À ces noms, j'aurais envie de rajouter ceux qui suivent : le traumatique, l'inlié, l'informe, l'angoisse automatique, la honte.

Aucun de ces noms n'arrive probablement à nommer exactement ce qui, justement, échappe au nom tout en l'appelant. Ces noms essayent de donner un nom à une ou à des expérience(s) vécue(s) originaire(s) dont les traces sont là en nous, non représentées, se présentant dans un état de non-liaison. Ce que nous redoutons, plus que tout, est de nous retrouver, à nouveau, dans cet état où règne l'absence que je qualifierai, cette fois-ci, à la suite de Freud d'absence de liaison. Pour éviter le retour de cet état, l'humain a inventé cette aptitude au transfert. De celle-ci, nous ne disposons pas tous de manière identique ou égale. L'aptitude au transfert est en puissance en chaque humain, mais elle ne devient effectivement actuelle que dans la rencontre de l'indispensable objet. 
La difficulté à garder le cap de mon propos est l'expression de ce que J.-L. Donnet a désigné comme l'inévitable écart théorico-clinique 12 ou de ce que J. Cl. Lavie souligne lorsqu'il écrit : "Les psychanalystes pourtant, à l'instar de tout un chacun, ne peuvent éviter de se comporter eux-mêmes comme s'ils savaient maîtriser ce qu'ils disent au présent de leur parole. C'est que la révélation de Freud ne donne pas pour autant le pouvoir de se soustraire à ce qu'elle dénonce. Cela veut dire que, pour l'esprit humain qui les promeut, il est des vérités qui ne peuvent que rester mythiques, leur réalité restant déniée dans cela même qui constitue leur approche ${ }^{13}$."

C'est de l'oubli momentané du Schreck qu'elle permettrait que l'ange, comme figure esthétique, tirerait son pouvoir de séduction. Nous ne devrions pas méconnaître que l'étude du phénomène de la séduction loin de "lever un voile sur les débuts de la pulsion sexuelle " vient trop souvent en "brouiller la vision que nous en avons ». La figure de l'ange traduirait notre volonté d'ignorer ce à quoi le passage de l'ange nous a éveillé. Face à notre aptitude à être séduit et à séduire (est-ce la même aptitude que celle au transfert ?), le grand avantage de l'objet est qu'il nous permet de mettre à la périphérie par projection (dans les zones érogènes, dans le moi, dans les objets aimés ou haïs et dans tout ce qui peut être imaginé) une partie de ce qui, d'abord, se présente comme "une démangeaison ou une stimulation d'origine centrale ", endogène. Par ce moyen peut être méconnue l'existence "d'une pulsion qui, par nature, ne manifeste pas tant que ça un besoin d'objet 14 ».

Ce que je viens de dire me rappelle une devinette. Quelle est la différence entre un chien et un homme ? Un chien se gratte toujours là où ça le démange, un homme presque toujours ailleurs. À la façon dont je me gratte, je crois qu'il n'y a aucun doute, je suis bien un homme, même s'il reste fort problématique de dire ce qu'est l'homme. Et ce n'est pas Edipe qui me démentira. Lui qui, sur son déclin, sorti de sa royauté, devenu cet exilé dont Colone sera le dernier asile, accepta, enfin, de supporter l'énigme en déclarant : «quand je ne suis plus rien, alors je suis un homme ». Cessant alors de vouloir être ce à quoi il voulait croire qu'il ressemblait, ne se prenant plus pour Edipe-Roi, il put enfin voir, au-delà de la ressemblance, la figure de l'homme en lui. Edipe à Colone touche certainement à ce point d' $\mathrm{d}^{\prime}$ originale indétermination » dont parle R. Antelme. Il le désigne comme ce point d'où "on pourrait observer de près » la formation d'un homme, " en faisant la part du

12. J.-L. Donnet, "L'écart théorico-clinique ", dans Le divan bien tempéré, Paris, PUF, 1995.

13. J.Cl. Lavie, "Les mots en jeu ", préface à Le mot d'esprit et sa relation à l'inconscient, trad. D. Messier, Paris, Gallimard, 1988, p. 23.

14. Les différentes citations de ce paragraphe proviennent de S. Freud, Trois essais sur la théorie sexuelle, trad. Ph. Kœppel, Paris, Gallimard, 1987, p. 119. 
caractère morbide de la formation ». Cette lettre du jeudi 21 juin 1945 que Robert Antelme écrit à son ami Dionys Mascolo est tellement terrible qu'elle sera oubliée pendant plus de quarante ans et que, la retrouvant, Mascolo se verra contraint d'écrire Autour d'un effort de mémoire 15 sans pouvoir achever le travail de remémoration. Le danger auquel Antelme se sent exposé, à ce moment-là, où il reprend pied après être revenu de Dachau, « c'est, écrit-il, de croire à ma ressemblance avec moi-même ». Un espoir éclaire sa plume : "Peutêtre, j'accepterai la ressemblance avec moi-même parce que je saurai qu'elle n'est pas. » Comment pourrait-on lire, sans trembler, des phrases telles que : "Dans l'enfer, on dit tout, ce doit d'ailleurs être à cela que nous, nous le reconnaissons ; pour ma part, c'est surtout comme cela que j'en ai eu la révélation. Dans notre monde au contraire on a l'habitude de choisir et je crois que je ne sais plus choisir. Eh bien, dans ce qui chez d'autres représentait pour moi l'enfer, tout dire, c'est là que j'ai vécu mon paradis."

« FAIRE » L'HUMAIN, « FAIRE » LE PSYCHANALYSTE

Revenons à mes raisons de rester dans les parages de l'ange et de proclamer mon humanité. Je n'ai jamais, pas plus que le Johnny que chante Piaf, été un ange, en tout cas pas à ma souvenance. Si nous admettons que nous n'avons jamais été des anges, cela veut dire que nous n'avons même pas eu la chance d'une quelconque déchéance. N'en déplaise à Morel et à sa thèse de la dégénérescence avec laquelle nous n'avons pas encore véritablement fini d'en finir, il n'y a rien de cassé en l'homme, il n'est pas incomplet. Par contre, son inachèvement, sa néoténie, sa dépendance, son peu de ressources autoconservatrices propres et la durable persistance en lui de caractères liés à un infantilisme constitutionnel, tout cela existe, non comme un défaut mais en tant que spécificité de l'espèce, qui ne l'a pas handicapée puisqu'elle est devenue la ressource de sa survie, de son expansion et de son apparent hégémonisme écologique.

Il n'y a donc pas grand-chose à réparer chez l'homme, pas grand-chose à restaurer. Et pas, autant de choses à retrouver que le croit le Moi. Il y a encore beaucoup de choses à construire et existent surtout des constructions déjà faites qu'il n'est peut-être pas inutile d'analyser, de déconstruire, de décomposer pour en repérer les différents éléments qui sont moins solidaires entre eux que ne le voudrait notre incorrigible propension à la synthèse.

Nous rêvons d'être asymptomatique, alors que nous ne pouvons être qu'asymptotique. Mais, c'est que nous avons beaucoup de mal à rompre avec la méde- 
cine - non pas celle d'Hippocrate - mais celle qui pense l'homme-machine ou neuronal. Nous refusons de penser que "les névroses ont justement une fonction biologique en tant que mesures défensives et une raison d'être sociale 16 ». Nous avons du mal à penser ces affections (et ce sont bien des affections déterminées par certaines affinités électives) autrement que comme des maladies.

Je vais en finir avec la première raison de m'être attardé auprès de l'ange. Il m'est parfois arrivé de jouer à l'ange, mais, vous savez tous, que «le malheur veut que qui veut faire l'ange fait la bête 17 ». Écoutons ce que Montaigne nous dit à ce propos : "Ésope, ce grand homme vit son maistre qui pissoit en se promenant: quoy donq, fit-il, nous faudra-t-il chier en courant? Mesnageons le temps; encore nous reste-t-il, beaucoup d'oisif et mal employé. Notre esprit n'a volontiers pas assez d'autres heures à faire ses besongnes, sans se désassocier du corps en ce peu d'espace qu'il lui faut pour sa nécessité. [Les hommes] veulent se mettre hors d'eux et échapper à l'homme. C'est folie : au lieu de se transformer en anges, ils se transforment en bêtes; au lieu de se hausser, ils s'abattent ${ }^{18}$. "

Se transformer en bête n'est pas accepter d'être l'animal que nous répugnons à être. La volonté d'ignorer est l'une des passions humaines qui menace le plus l'humanité, nous nous évertuons, en ne voulant pas savoir ce que nous désirons, à vouloir d'autres conditions que l'humaine condition.

Est-il possible de sortir de l'Edipe, de vivre son déclin et de parvenir à Colone ? Comment faire l'humain ? Qu'est-ce que faire l'humain ? Comment ça se fait humain. Bien que nous soyons d'accord avec la déclaration de Freud à Binswanger : "Il n'est rien dans la structure de l'homme qui le prédispose à s'occuper de psychanalyse 19 ", nous savons qu'il arrive pourtant de pouvoir faire de l'analyse. Ce que j'essaye de penser c'est comment je fais l'analyste. Je reprends, ici, la manière italienne de dire que je préfère à l'affirmation française : je suis analyste. Autrement dit : comment un humain peut-il faire l'analyste pour faire que ça se fasse un peu plus humain sans que, pour autant, l'humain n'efface ce qui de lui ne cesse pas de ne pas prendre figure humaine?

Je ne pense pas qu'il soit impossible de faire ce métier, pas plus d'ailleurs que ceux de gouverner, d'éduquer ou d'élever des enfants. Ce n'est certes pas facile, cela peut-être usant et c'est parfois désespérant. L'impossible dans ce métier n'est pas de le faire, c'est que, pour le faire, il faut supporter l'impossible, celui qui est en relation avec le nécessaire. Or, pour supporter cet

16. S. Freud, « Perspectives d'avenir de la thérapeutique analytique ", trad. A. Berman, dans $L a$ technique psychanalytique, Paris, PUF, 1977, p. 33.

17. B. Pascal, Pensées, OC. II, 2000, 112, 468, 572, Paris, Gallimard, coll. « Bibliothèque de la Pléiade », 2000.

18. Montaigne, Essais III, XIII, 1256, Paris, Gallimard, coll. « Bibliothèque de la Pléiade », 1961.

19. S. Freud, L. Binswanger, Correspondance 1908-1938, Paris, Calmann-Lévy, 1995, p. 134. 
impossible, pour ne pas lui tourner le dos, pour ne pas nous précipiter comme séducteur ou comme agresseur sur un objet qui serait à portée de main, il est nécessaire d'habiter sa haine. Et quand on n'y parvient pas, il faut écouter R. Char nous dire qu'il plaint "celui qui fait payer à autrui ses propres dettes en les aggravant du prestige de la fausse vacuité $20 »$.

Nous ne supportons pas que le retrait de l'ange nous laisse dans un monde certes interprétable pour partie, mais dont l'interprétation n'est pas Révélation, juste un moment dans un chemin de construction. "Sommes-nous voués à n'être que des débuts de vérité 21 ?", se demande R. Char. Oui, et nos interprétations ne nous permettent d'habiter le monde qu'avec un certain malaise, qui résulte du fait que nous sommes mal à l'aise avec ce qui nous habite et que nous ne nous décidons pas toujours à habiter.

«MON » ANGE

Je ne vais pas encore quitter l'ange, car je n'ai pas encore parlé de ma seconde raison de l'accompagner. Il est remarquable qu'il soit plus facile, à la plupart d'entre nous, d'essayer de faire l'ange et donc la bête, que d'accepter d'être pris par l'autre pour "son » ange. Quand l'être aimé se met à vous appeler « mon ange », cette déclaration est rarement prise pour argent comptant et elle n'est pas sans susciter une certaine méfiance, voire un mouvement de rejet. Avez-vous remarqué comment dans les affaires amoureuses, nous pouvons parfois devenir de terribles ergoteurs qui n'en finissent pas de demander des explications? "Quand il dit qu'il m'aime, disait une patiente, je ne me reconnais pas dans celle dont il parle. » En amour, on a l'impression, assez souvent, soit que chacun demande sans cesse à l'autre s'il est bien sûr qu'il n'y a pas erreur sur la personne, soit qu'il passe beaucoup de temps à se demander s'il ne s'est pas trompé, est-ce qu'il l'aime vraiment. Ne croyons pas que ça fasse perdre tant de temps que ça, parce qu'au moins cette préoccupation occupe l'esprit et le détourne de tant d'autres choses... plus inquiétantes peut-être.

En dehors du côté tragiquement drôle que cela peut avoir, il me semble qu'il y a quelques intérêts à s'arrêter sur cette histoire d'être pris par un autre pour «son » ange et notre « légère » (c'est un euphémisme !) difficulté à y croire, malgré l'envie féroce que nous aurions aussi que cela soit vrai. Quand l'autre nous appelle " mon ange », ne devrait-on pas se résoudre à concéder

20. R. Char, «Feuillets d'Hypnos ", dans CEuvres complètes, Paris, Gallimard, coll. «Bibliothèque de la Pléiade », 1983, p. 216.

21. R. Char, ibid. 
que cela n'appelle nulle discussion. Il faut supporter d'entendre ce qui est dit et l'accepter comme étant terriblement vrai.

Par l'indispensable objet, nous touchons à une vérité qui ne saurait que se dérober à toute saisie et, devant laquelle, un mouvement de recul peut nous amener à faire retraite dans l'objet : il tend alors à venir en lieu et place de la Vérité jusqu'à pouvoir devenir bien plus important qu'elle. Peut-être que le plus terrible quand quelqu'un que vous aimez vous appelle « Mon ange », c'est de devoir concéder que, non seulement il est probablement juste que vous en êtes un et, même peut-être, vraiment son ange, au-delà de tout ce que vous pouvez imaginer, car par « votre » truchement, cet être s'est retrouvé mis en présence de l'ange. Mais que vous soyez « son » ange ne fait pas pour autant de vous un ange. Or, c'est cet écart-là qu'il faut pouvoir accepter de supporter, sans vouloir l'abolir ou l'ignorer. Au point même de la plus extrême proximité, de l'intimité la plus grande avec un autre que soi se laisse entendre une distance qui fait de chacun l'éloigné de l'autre, dans un éloignement qui déjoue la " foule à deux » que tendent à former les amants. Il existe, à la fois, l'effet de masse de la foule et l'échec de cette tendance qui fait apparaître non pas une seule scène psychique pour deux, mais deux scènes séparées. Sur chacune se pratique un traitement psychique du psychique par le psychique (celui de l'autre et le sien) et une action psychique sur le psychique de l'autre.

Il faut, par ailleurs, convenir que nous n'avons pas complètement tort de nous méfier quand nous sommes trop rapidement et avec trop d'empressement, désignés comme «mon ange ». Vous étiez «mon ange » et il ou elle était "mon ange ». Des choses qui, jusque-là, paraissaient difficiles, pénibles voire impossibles devenaient possibles. Vous sentiez que des ailes, d'ange bien sûr, étaient en train de vous pousser. Et puis, imperceptiblement, insidieusement vous, il ou elle n'était plus " Mon ange ». Parfois, vous, il ou elle était même devenu le «diable », le « démon ». Notons qu'envers cet être intermédiairelà, nous ne manifestons qu'assez peu de possessivité et que nous tentons plutôt de l'expulser, de le mettre au dehors, pas dedans. Et, de manière assez perturbante, souvent assez ravageuse, de l'amour, on est passé à la haine. L'amour, qui semblait premier, se serait transformé secondairement en haine.

Mais, il est nécessaire de procéder à un retournement logique. Ce qui survient apparemment en second, parfois assez tardivement, au moment où la cure semblait prête à se conclure, cette haine passionnellement haineuse ou amoureuse est-elle vraiment seconde ? N'est-elle pas première et, ce qui, dans la cure, s'est manifesté en premier, le positif du transfert, n'avait-il pas pour fonction de refouler ce dont la levée du refoulement exigera beaucoup de patience, pas mal de doigté, un grand tact et une endurance peu commune des deux partenaires de l'entreprise analytique? 
Le premier mouvement est celui-là même qu' Augustin, qui fut un Saint pas un ange, décrit si bien dans les Confessions :

"C'est que l'on aime tant la vérité que, s'ils [les hommes] aiment autre chose $q u$ 'elle, ils veulent que la vérité, ce soit précisément l'objet de leur amour; et, comme ils ne sauraient accepter de se tromper, ils n'admettent pas qu'on leur démontre leur erreur. Voilà pourquoi ce qu'ils aiment à la place de la vérité leur fait haïr la vérité même. Ils en aiment la lumière, mais en détestent les reproches; Acceptant de tromper, mais non de se tromper, ils l'aiment quand elle manifeste ce qu'elle est, mais la hä̈ssent quand elle manifeste ce qu'ils sont. Telle sera sa sanction: ils ne veulent pas qu'elle les démasque; elle les démasquera malgré eux, mais gardera pour eux le visage masqué ${ }^{22}$. »

Tout au long de mon écriture, j'aurai espéré échapper à la tentation dont parle Augustin, sans y parvenir véritablement. Tout au plus puis-je espérer, avoir soutenu le paradoxe. Je n'ai toujours pas dit ce que j'appelle Vérité. Qu'est-ce que cette Vérité dont je vous parle ? Pour être tout à fait honnête (c'est le genre de déclaration dont on doit se méfier), je ne sais pas ce qu'est la Vérité.

Mais, il me faut cependant essayer d'en dire quelque chose. La Vérité, $c^{\prime}$ est que notre inachèvement est insupportable ; c'est que le monde n'est pas fait pour nous, pas plus que nous pour le monde ; $c^{\prime}$ est qu'il n'est pas fait non plus contre nous, ni nous contre lui ; c'est qu'il y a quelque chose dans la nature même de la pulsion sexuelle qui ne se prête pas à la pleine satisfaction ; c'est que quelque soit notre capacité psychique à traiter les excitations, à les lier à des représentations, il y a toujours du reste non traité, intraitable.

\section{LA HONTE DE L'ÉCONOMIQUE OU LES DANGERS DE L'ANALYSE}

J'ai un peu honte de n'avoir à faire entendre que des choses assez banales. C'est étonnant les moments où l'on a honte. J'ai, par exemple, été toujours surpris par ce passage $\mathrm{d}^{\prime}$ " Analyse avec fin et analyse sans fin » où, étonnamment, Freud déclare qu'il a honte. Cela survient alors qu'il est obligé de constater la "puissance irrésistible du facteur quantitatif dans la causation de la maladie ». Ce facteur, présent dès le départ, on ne peut pas le faire disparaître, on ne peut que le dompter partiellement et toujours bien moins qu'il n'a semblé dans un premier temps. Mais, de plus, son influence et sa force se voyant, avec le temps, immanquablement renforcées, Freud avance qu'il

22. Saint Augustin, Les Confessions, Livre X, «La mémoire des traces », XXIII, 34, trad. P. Cambronne, dans CEuvres I, Paris, Gallimard, coll. «Bibliothèque de la Pléiade », 1998. 
serait plus juste de qualifier la force pulsionnelle d'actuelle plutôt que de ne souligner que son caractère constitutionnel. Et là, Freud écrit cette chose étrange :

"J'ai ici l'impression que je devrais avoir honte de toutes ces discussions pesantes, ce qu'elles disent étant connu depuis longtemps et allant de soi. Effectivement nous nous sommes toujours comporté comme si nous savions cela, à cette réserve près que dans nos représentations théoriques nous avons négligé la plupart du temps de tenir compte du point de vue économique dans la même mesure que des points de vue dynamique et topique. Mon excuse c'est donc que je remets en mémoire cette négligence ${ }^{23}$. »

Le moment de honte, n'est-ce pas ce moment où, aussi accroché soit-on à l'objet que l'on prend pour la Vérité, la puissance de l'économique défait toutes les constructions de secours que nous avions édifiées pour la dompter, la civiliser et où survient la commotion que nous avions attribuée au trop de présence de l'Ange ? Surgissent alors les états d'angoisse automatique, de honte, qui sont deux états d'affect originaires ${ }^{24}$. Je soutiendrai, ici, que la haine est le mouvement par lequel on surmonte l'angoisse et la honte en créant notre duplice réalité : l'une que l'on nomme intérieure et l'autre extérieure - sans que l'on puisse distinguer de manière assurée que, là, est l'extérieur et, ici, l'intérieur.

Sachant par expérience quelles résistances se dressent sur la voie qui mène à l'au-delà du principe de plaisir, nous ne pouvons que nous questionner sur ce qui peut amener quelqu'un à s'exposer si régulièrement dans la pratique d'analyste au retour de cet au-delà. Nous ne devrions pas être surpris de devoir reconnaître que, plus d'une fois, plane sur la cure la menace d'un mésusage du transfert par la personne de l'analyste. Celle-ci, ne voulant pas se voir attaquée par la remise en cause du principe de plaisir, vient à s'opposer à ce que s'effectue la fonction d'analyste. Je tiens à préciser que l'analyste n'est pas tant attaqué par le patient (identification projective, effort pour rendre l'autre fou, etc.) que par ses propres motions pulsionnelles dont la force actuelle est renforcée en raison du commerce incessant entretenu avec tout le refoulé, qui, dans l'âme humaine, lutte pour sa libération.

Dans la situation analytique, la fonction analytique risque de ne pas pouvoir être assumée quand la personne de l'analyste doit faire face à toutes

23. S. Freud, " L'analyse avec fin et l'analyse sans fin ", trad. J. Altounian, A. Bourguignon, P. Cotet, A. Rauzy, dans Résultats, idées, problèmes, II, 1921-1938, Paris, PUF, 1985.

24. Sur la honte, je renverrai aux travaux entre autres de C. Barazer, «Hontes sans issues », dans Documents et débats, Bulletin de l'Association psychanalytique de France, $\mathrm{n}^{\circ} 52$ et « Ulysse nu et couvert de boue ", dans Revue française de psychanalyse, t. LXVII, n 5, Paris, PUF, 2003. 
les revendications pulsionnelles qu'elle peut habituellement maintenir dans l'état de répression et qui, du fait de la cure, risque ici et maintenant de se voir arrachées au sommeil. Ce sont les "dangers de l'analyse », nous dit Freud, en précisant qu'ils ne menacent pas tant le «partenaire passif » de la situation analytique (c'est ainsi qu'il nomme le patient) que le « partenaire actif » (terme dont il désigne l'analyste). Pour cette raison, Freud estime que l'analyste devrait périodiquement, tous les cinq ans par exemple, «se constituer à nouveau objet de l'analyse sans avoir honte de cette démarche ». À nouveau, ici, ressurgit, effet de l'économique, la question de la honte.

Cette attaque de la personne est difficile à supporter parce qu'il est indéniablement difficile d'accepter de cesser de "ressembler à moi-même », de perdre momentanément tout ressemblance à l'objet que nous voudrions être ou avoir. Nous sommes toujours prêt, "un soir de demi-brume à Londres » ou ailleurs : ici et maintenant par exemple et, de manière vérifiée, dans la cure, à nous prendre pour cet objet ou à prendre un autre pour cet objet. Il suffit que cet objet-là paraisse à la semblance soit de mon bel amour, soit du bel amour que nous voudrions être pour l'autre. Et, à ce moment-là, moment de transfert, le ressemblant tend à être traité comme la chose même.

L'exigence que nous redevenions objet de l'analyse ne fait que souligner que rester analyste ne saurait être acquis une fois pour toutes et que nous ne parviendrons jamais complètement à nous rendre ami avec ce qui, bien que nous fondant, ne cesse d'être méconnu comme notre fondement. Bizarre, donc, que de vouloir occuper cette place d'analyste ! Nous pourrons toujours arguer hérö̈quement de la force de notre amour de la vérité. À condition de ne pas oublier la remarque d'Augustin, cela ne serait sans doute même pas faux. Notre amour de la vérité s'avère aussi être notre haine de celle-ci dès qu'elle contrarie nos amours selon le principe de plaisir. La domination de ce dernier se révèle dans la passion d'ignorer tout ce qui le contrarie et de rendre non advenu tout ce qui le perturbe.

L'histoire de la formation analytique étant ce qu'elle est, nous ne pouvons pas ignorer que lorsque la plupart d'entre nous ont commencé leur pratique d'analyste, leur amour de la vérité n'avait sans doute pas encore atteint la force - mais peut-elle être atteinte ? - qui leur aurait permis de renoncer aux bénéfices secondaires que permet la névrose de transfert. On pourrait, comme cela s'entend assez souvent dans les conversations entre collègues, dire qu'il n'est pas exclu que certains d'entre nous ne soient devenus analystes que par refus « de guérir » de la névrose de transfert. Cela aurait été un moyen d'échapper, pour partie, à la reconnaissance tant des exigences de la culture que de la réalité effective de la puissance de certaines motions pulsionnelles. Je ne dis pas que certains analystes sont mal analysés. C'est, certes, une critique que l'on entend plus d'une fois dans la bouche d'un col- 
lègue à l'égard d'un autre et c'est aussi ce qu'exprime le constat légèrement mélancolique que " notre » propre analyse n'aurait pas été assez loin... du fait, bien sûr, des limites de notre analyste dont l'inanalysé nous aurait empêché d'aller plus loin. L'idée du " mal analysé » me paraît relever plus de l'idéal asymptomatique que de la reconnaissance de la réalité asymptotique de la vie psychique. Freud d'ailleurs dit que, plutôt que de parler d'analyse non-terminée, il serait judicieux de parler d'analyse inachevée - et l'inachèvement, dont il est question, est un caractère inhérent au processus analytique lui-même 25 .

Dès les débuts de la psychanalyse, a été dénoncé le risque que constitue, du côté de l'analyste, les effets sur la cure des restes transférentiels non résolus dans sa propre analyse. Ferenczi a ouvert cette critique en reprochant à Freud de ne pas avoir suffisamment analysé le transfert négatif. Il a cru trouver, dans la mutualisation de l'analyse, le moyen de surmonter cette difficulté. La question était et reste hautement pertinente, même si ce n'était pas la voie pour y répondre. Ce qui est sûr, c'est qu'en ce point l'analyste, au lieu d'être accoucheur, peut devenir faiseuse d'ange : c'est l'avortement de l'infantile qui aura alors lieu.

\section{PARADOXE DU TRANSFERT}

Tout transfert, qu'il soit positif ou négatif, n'est-il pas justement, avant tout, la tentative du maintien du refoulement et, particulièrement, du refoulement du caractère primitivement haineux de la pulsion sexuelle ? Le paradoxe du transfert, c'est que lorsqu'il se déploie sous sa forme positive, il facilite certes la remémoration, mais les souvenirs produits peuvent devenir des objets que l'on offre à l'analyste dans l'espoir d'obtenir la satisfaction des désirs infantiles et ils s'avèrent, alors, être un véritable obstacle au travail de remémoration et, surtout, à celui de la perlaboration. Chaque souvenir produit porte la menace d'être le lieu de la constitution ou du renforcement d'une fixation libidinale (le chemin devient alors le but). Se souvenir devient ainsi une répétition en acte dans le transfert qui, s'opposant à la remémoration, empêche la levée du refoulement et évite la rencontre de l'amnésie infantile structurelle qui nous contraint au travail de la construction. Il y a un autre paradoxe dans le transfert, c'est que lorsqu'il devient négatif, il menace de détruire toutes les conquêtes qui paraissaient avoir été faites et que la cure risque d'être prématurément interrompue. Mais, s'il peut être analysé ce qui se joue là, existe la possibilité d'une réelle efficience dans le temps des effets

25. F. Villa, « La personne du psychanalyste : obstacle à la remémoration, une résistance à accéder à l'au-delà du principe de plaisir ", dans Cliniques méditerranéennes, 67-2003, p. 172-190. 
de l'analyse. Ainsi peuvent être obtenues de réelles modifications du moi, y compris de son caractère (effet d'une détransposition psychique des pulsions) 26 .

À quoi donc nous offrons-nous en appelant par notre silence inaugural le patient au transfert ? Oserions-nous soutenir que c'est à la haine pulsionnelle, celle du patient et la nôtre ? J'en doute, il est plus probable que nous espérons plutôt pouvoir lui échapper. C'est dans notre capacité à supporter que nous ne pouvons pas lui échapper et à le reconnaître que gisent les chances des quelques résultats qu'on peut escompter de la psychanalyse.

Ce que l'on appelle improprement contre-transfert, parce que, justement, il ne va pas contre le transfert, il ne pousse pas vers son analyse, mais au contraire vers le renforcement du transfert comme inanalysable, n'est que la manière dont nous sommes pris par la tendance à oublier que nous ne sommes pas le véritable interlocuteur du patient ni le destinataire de sa parole. Nous ne sommes que le support par où la parole du patient peut prendre sens en découvrant l'interlocuteur dénié et les souhaits infantiles qui trouvent satisfaction dans la déformation du transfert. Le transfert du patient, en nous instaurant comme l'un des objets d'une série qui préexiste à la rencontre : un objet à la semblance de ses objets, nous déstabilise parce qu'il révèle notre répugnance à être un objet qui ressemble tellement peu à ce que nous croyons ou voulons être. Le moi de l'analyste est, alors, tenté, en expulsant cette perturbation, de se refermer sur sa propre série d'objets (dont le moi fait partie). Le mouvement où se manifeste notre propre tendance au transfert est celui-là même qui, si nous surmontons notre répugnance à le faire, rend possible l'analyse du moi qui résistera pourtant à sa décomposition. Arrêter ce mouvement peut devenir l'enjeu moïque dominant l'analyste. Il peut se saisir de la fausse connexion du transfert en la surdéterminant d'une fausse connexion qui lui est propre. Sa personne se tient alors, crédulement, hypnotiquement (effet de foule) dans la position d'être devenu le dépositaire, le destinataire d'une parole affective puissamment réveillée par la cure, mais qui est adressée du lieu de l'infantile à un personnage préhistorique. L'analyste n'est pas alors la «troisième personne » qui permet, comme dans Le mot d'esprit, qu'émerge l'interlocuteur de l'adresse haineuse ou obscène. L'analyste prend alors la place du séducteur, du führer.

En ce point, il nous faut revenir sur ce que nous avons appelé plus haut le projet. Ce que nous nommons ainsi est une radicale tentative d'expulsion

26. F. Villa, « Le caractère dans la théorie freudienne : une "sous-espèce de sublimation " dans les transpositions pulsionnelles », dans S. Dreyfus ; J. Bouhsira ; A. Fine, Caractère(s), Monographie de la Revue française de psychanalyse, Paris, PUF, 2004. 
de l'excitation au-dehors. Un dehors qui n'est pas constitué, pas plus que le dedans d'ailleurs. Ils vont se constituer dans ce moment où l'excitation ressentie va être « reconnue » comme devant être mise dehors. L'expulsion aura partiellement lieu et la partie expulsée créera la réalité extérieure en rencontrant un objet. Celui-ci, selon la manière dont il interceptera ce qui le prend comme support, permettra, en disposant le monde autrement, soit le retour vers l'intérieur (l'expulseur pourra alors effectuer un travail de réappropriation de l'expulsé qui fut antérieurement désappropriant), soit, le retour ne pouvant avoir lieu, la projection s'accentuera radicalement. Mais, dès l'origine, il s'avèrera qu'une part de l'excitation n'est pas expulsable, c'est celle qui est au fondement de l'introjection ferenczienne. La projection complète échouant, on peut imaginer que ce qui ne peut être projeté et doit être introjecté se voit fragmenté en différentes parties d'où naîtra la topique 27 .

\section{HABITER LA HAINE, SORTIR DE LA PSYCHOLOGIE DE FOULE}

Ce mouvement expulsif du « projet » est mouvement de pure haine d'une haine sans objet, déterminée uniquement comme mouvement de rejet. C'est dans ce mouvement que sera créé l'objet et c'est cela qui permet de dire que le premier lien à l'objet est la haine, un lien d'autant plus solide qu'il connaîtra un renforcement à l'abri du refoulement. Le silence qui, nous l'avons dit, est propice au retour du refoulé, est le lieu où peut nous être donnée la capacité d'habiter la haine sans l'agir dans le monde pour s'en débarrasser. C'est pour cela que la décision de sortir du silence, ou d'y persister, se révèle être un moment décisif dont dépend la levée, le maintien ou le renforcement du refoulement de la haine. De même, c'est un moment essentiel, pour la perlaboration, qu'après avoir parlé, nous supportions de nous retirer dans le silence pour découvrir et supporter les effets de la parole - les effets de s'être entendu dire autre chose que ce que l'on croyait vouloir dire, les effets d'entendre de quelle oreille le patient a entendu notre parole. L'enjeu est, ici, essentiel, puisqu'il est celui de l'infini travail d'individuation auquel tant l'analyste que le patient sont voués. L'enjeu est que le patient, en entendant soit notre silence, soit notre parole, puisse réinscrire ce dont il souffre dans l'histoire de sa vie, dans celle d'une famille, d'une cité, d'un peuple, de l'humanité, de la vie.

27. Ce point de vue éclairerait le caractère paradoxal de surmoi qui tout en représentant les exigences du monde externe reste au plus près des exigences du ça. L'idéal du moi, qui est le noyau du surmoi, se constituerait à partir du non-expulsable et serait dans une relation étroite au ça. Ce n'est que dans la constitution ultérieure du surmoi (par introjection des images parentales et des exigences culturelles) que le lien à la réalité extérieure se verrait renforcé. 
Ce que nous appelons habiter la haine est une façon de désigner la sortie de la psychologie de foule (le lien qui la fonde étant le refoulement de la haine) pour passer à la psychologie individuelle qui et que permet le processus d'individuation. Nous savons que Freud attribue au poète épique d'avoir accompli ce pas. Il l'accomplit en créant le mythe où apparaît le héros qui prétend avoir accompli, seul, l'acte que la horde, comme masse indifférenciée, avait perpétré. Le poète, pour faire ce pas, doit d'abord se retirer, se détacher de la masse, pour ensuite trouver, dans la réalité effective, le chemin du retour à elle. Ce retour est une avancée vers la foule, pour parler aux autres de cette distance que créé le fait de se poser comme auteur de l'acte qu'ils avaient fait tous ensemble. Il cesse de vouloir ignorer le meurtre commis. Il devient responsable individuellement de ce que la horde a fait, et ce faisant, il devient un homme de culture et cesse d'être, momentanément, un hypocrite de la culture. Certes, comme le dit Freud, quant « au fond, ce héros n'est nul autre que » le poète mais, il l'est au prix du déplacement du narrateur vers un héros dont il invente la figure à partir du fond et non d'une ressemblance formelle avec lui-même. Il ne peut le faire qu'en s'abaissant jusqu'à la réalité déniée par le moi, en rabaissant les prétentions du moi à l'idéalisation. Touchant au fond commun, où la vie psychique ne prend son essor que d'avoir touché au mort que l'homme a lui-même produit par le meurtre dans son premier mouvement de haine, le poète permet aux autres de reconnaître la même relation de désirance au Père originaire et, par la fantaisie, il rend possible de s'élever au-dessus de la culpabilité et de la simple contrainte de l'obéissance après-coup.

Parvenu en ce point, l'homme peut cesser d'être allongé et, touchant à l'horizon de sa verticalité, se mettre debout.

\section{BIBLIOGRAPHIE}

Assoun, P.-L. 2002. «De l'effrayant à l'effroyable. Figures freudiennes de Schreck», dans Apertura, $\mathrm{n}^{\circ} 17$, érès.

BARAZER, Cl. 1998. "Hontes sans issues », dans Documents et débats, Bulletin de l'Association psychanalytique de France, $\mathrm{n}^{\circ}$ 52, 2000.

BARAZER, Cl. 2003. «Ulysse nu et couvert de boue », dans Revue française de Psychanalyse, tome LXVII, $\mathrm{n}^{\circ}$ 5, Paris, PUF.

Char, R. 1946. «Feuillets d'Hypnos », dans CEuvres complètes, Paris, bibl. de la Pléiade, Gallimard, 1983.

DonNET, J.-L. 1984. « L'écart théorico-clinique », dans Le divan bien tempéré, Paris, PUF, 1995.

FÉDIDA, P. 1978. L'absence, Paris, Gallimard.

FERENCZI, S. 1909. "Transfert et introjection », dans Psychanalyse 1, CEuvres complètes : 1908-1912, trad. J. Dupont avec coll. de Garnier Ph. Paris, Payot, 1968. 
FREUD, S. 1896. «Esquisse d'une psychologie scientifique », dans La naissance de la psychanalyse, trad. A. Berman, Paris, PUF, 1956.

Freud, S. 1905. Trois essais sur la théorie sexuelle, trad. Ph. Kœeppel, Paris, Gallimard, 1987.

Freud, S. 1910. « Perspectives d'avenir de la thérapeutique analytique », trad. A. Berman, in La technique psychanalytique, Paris, PUF, 1977.

FreUd, S. 1937. «L'analyse avec fin et l'analyse sans fin », trad. J. Altounian, A. Bourguignon, P. Cotet, A. Rauzy, dans Résultats, idées, problèmes, II, 1921-1938, Paris, PUF, 1985.

Freud, S. ; Binswanger, L., 1995. Correspondance 1908-1938, Paris, Calmann-Lévy.

JeAN DE LA CRoIx. 1585. «Glose « a lo divino » », dans CEuvres complètes, trad. Par le P. Cyprien de la Nativité de la Vierge, Paris, Desclée de Brouwer, 1967.

LAVIE, J. Cl. 1988. "Les mots en jeu ", préface à Le mot d'esprit et sa relation à l'inconscient, trad. D. Messier, Paris, Gallimard.

Mascolo, D. 1987. Autour d'un effort de mémoire, Paris, Maurice Nadeau.

Montaigne. Essais III, XIII, 1256, Paris, bibl. de la Pléiade, Gallimard, 1961.

Pascal, B. Pensées, O.C. II, 2000, 112, 468, 572, Paris, bibl. de la Pléiade, Gallimard, 2000.

RILKe, R.M. Les élégies de Duino, trad. A. Guerne, Paris, Essais, Le Seuil, 1972.

Saint Augustin. Les Confessions, Livre X, "La mémoire des traces », XXIII, 34, trad.

P. Cambronne, in coll. La Pléiade, Euvres I, Paris, Gallimard, 1998.

VILLA, F. 2003. «La personne du psychanalyste : obstacle à la remémoration, une résistance à accéder à l'au-delà du principe de plaisir », in Cliniques méditerranéennes, 67, p. 172-190.

VILLA, F. 2004. «Le caractère dans la théorie freudienne : une « sous-espèce de sublimation » dans les transpositions pulsionnelles », in Dreyfus S. Bouhsira J. Fine A., Caractère(s), Monographie de la Revue française de psychanalyse, Paris, PUF.

Résumé

C'est plus d'une fois, que nous devons convenir, non sans une certaine haine, que Freud a quelques raisons d'écrire à Binswanger qu'il " n'est rien dans la structure de l'homme qui le prédispose à s'occuper de psychanalyse ». Et, c'est un pas de plus, coûteux psychiquement, que d'avoir à découvrir, dans le cours d'une cure, les mécanismes de défense que le moi secrète pour se soustraire aux effets de la psychanalyse.

Soutenir l'amour de la vérité (à entendre comme reconnaissance de cette réalité dont la psychopathologie ne cesse de montrer que l'humain est plus que disposé à s'en détourner) impose de surmonter la passion d'ignorer et d'habiter la haine. Le coût du travail psychanalytique est considérable, car il impose de surmonter des méconnaissances qui paraissaient jusque-là vitales (et qui l'étaient d'un certain point de vue) et de reconnaître la violence indomptable des motions pulsionnelles, les ressources de haine dont nous disposons et que le refoulement nous masque.

La pratique psychanalytique fait redécouvrir, à chaque fois comme une première fois, à quel point est présent et actuel en chacun le conflit entre « l'effort pour demeurer tel qu'on est » et l'effort pour se modifier en fonction des exigences de la réalité que le 
travail psychanalytique impose. C'est dans de tels moments que l'on prend la mesure de la difficulté, pour le patient mais aussi pour le psychanalyste, d'aller au-delà du principe de plaisir. La puissance d'emprise de celui-ci s'oppose alors à ce que le travail psychanalytique se fasse travail de culture et menace la cure de devenir un subtil moyen de continuer à se détourner de la réalité.

\section{Mots-clés}

Amour, effroi, haine, plaisir (principe de), psychanalyste, psychologie de foule, pulsion, remémoration, silence, transfert, vérité.

THE MISFORTUNES OF « OUR » LOVE OF TRUTH FACED WITH THE POTENCY OF THE PLEASURE PRINCIPLE

\section{Summary}

We have had to admit on more than one occasion, though not without a certain sense of hate, that Freud had good reasons to write to Binswanger that there is «nothing in man's framework that predisposes him to take an interest in psychoanalysis ». Then, during treatment, discovering the defence mechanisms that the ego secretes to evade the effects of the psychoanalysis takes matters a step further, though at considerable psychical cost.

Sustaining the love of truth (to be understood as a recognition of that reality whose psychopathology unfailingly shows us that human beings are ever inclined to turn away from it) requires us to overcome the passion of ignoring, and inhabiting hatred. The cost of psychoanalytical work is considerable as it requires us to overcome misconceptions that hitherto appeared to be vital (and which were so from a certain point of view) and recognise the uncontrollable violence of instinctual impulses, the resources of hate we have in us and that repression hides from us.

Psychoanalytical practice has us discover, ever as if for the first time, to what extent the conflict between « the effort to remain what you are » and the effort to change in relation to the demands of the reality the psychoanalytical effort imposes is present and of immediate concern in each of us. It is at such times that we take stock of the difficulties involved, for the patient but also for the psychoanalyst, in going beyond the pleasure principle. The latter's potency then stands in the way of the psychoanalytical work becoming a work on culture and threatens to make the cure become a subtle means of continuing to turn away from reality.

Keywords

Love, dread, hate, pleasure (principle), psychoanalyst, crowd psychology, drive, recall, silence, transfer, truth. 